\title{
PRIORITISATION OF HEALTH ISSUES AND STRATEGIES TO IMPROVE CHILD HEALTH
}

\author{
Garth Alperstein \\ Community Paediatrician, Community Health Services, \\ Central Sydney Area Health Service
}

\section{Victor Nossar}

Service Director, Department of Community Paediatrics, South Western Sydney Area Health Service

This article describes how Central Sydney Area Health Service, when developing a strategic plan to improve the health of children, prioritised health issues and determined interventions to address them.

To achieve better health outcomes for children, it is insufficient to document health needs and identify effective strategies to meet those needs. As all health services have finite resources, it is also necessary to prioritise services which are capable of achieving the maximum health gain. However, scant research has been published on the most appropriate means to establish priorities in health. The diagram by MacFarlane suggests some of the principles underpinning the prioritisation of health issues and interventions (Figure 1). ${ }^{1}$

In 1996, Central Sydney Area Health Service developed a strategic plan for children and youth to determine new services that should be established in the Area. ${ }^{2}$ The plan included consideration of MacFarlane's elements in determining the health issues that were a priority and the interventions to address them. Several different approaches were used to rate health issues to determine

\section{FIGURE 1}

\section{SOME OF THE BASES OF PRIORITISATION OF HEALTH ISSUES AND INTERVENTIONS(FROM MACFARLANE ${ }^{1}$ )}

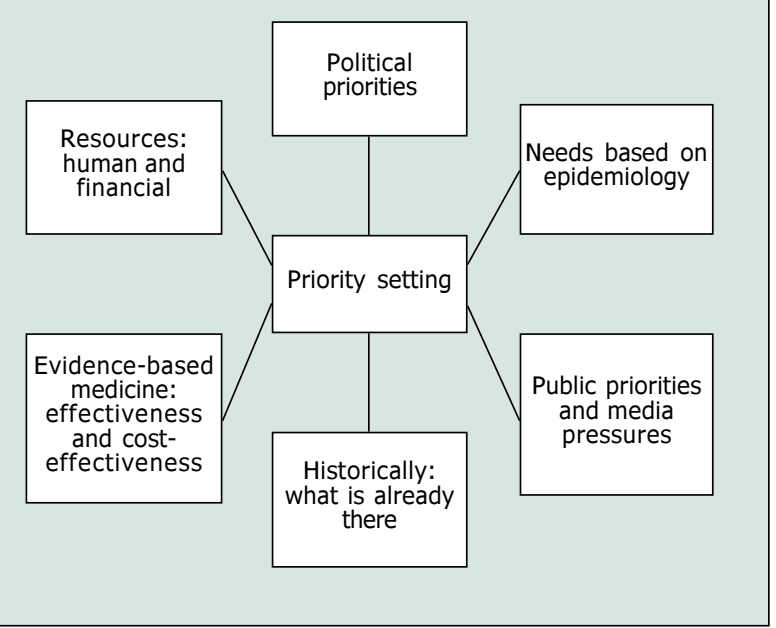

their relative importance and the ability of interventions to achieve health gain.

Criteria used to rank health issues included:

- the numbers of people affected by the particular health issue: that is, the size of the problem in the community (the number of people affected was estimated from either prevalence or incidence data, as appropriate)

- the mortality resulting from the health issue

- the morbidity resulting from the health issue; that is, the effect of the condition on the affected person's ability to function independently. The effect on the duration and quality of life was measured with a scale that ranged from occasional visits to the doctor or primary care provider to severe disablement or death.

Further criteria included:

- comparison with NSW rates of incidence and prevalence

- comparison with national targets, where available. ${ }^{3}$

Several numerical scoring systems, with different weights for each of the variables identified (incidence or prevalence, severity, mortality, comparison against NSW rates and against national targets), were given a trial to determine the most objective method for prioritising issues on the basis of the described criteria. Different mathematical methods were also tried. The results proved to be remarkably consistent for the different trials, and the health issues remained in a similar order, forming consistent high-, medium- and low-priority groupings.

A less numerical scoring system, described by Morley in 1973, was also given a trial. ${ }^{4}$ This system was based on four criteria:

- prevalence of the condition

- severity of the condition

- availability of effective intervention

- community concern about the health issue.

This system drew on prevalence rates, functional status scores, research on interventions, and community consultation to establish comparisons. (Consultations had been held with eight different community groups, with one to three meetings for each group.) This approach combined the burden of suffering with the availability of effective interventions and community concern into a single scoring system.

Once again, regardless of the approach used, the grouping of the health issues into high, medium and low priority remained broadly consistent.

The final priorities established by the Central Sydney Area strategic plan were the result of the methodologies described, but also drew on local additional community 
consultations, discussion with relevant experts and common sense (Table 1).

Socially and environmentally related issues proved difficult to quantify, but from both the community consultations and discussions with relevant experts, there appeared to be broad consensus that these issues belonged in the high-priority group.

This process identified both the issues and the strategies that could produce health gain for the child population of Central Sydney. It is important to recognise that many of the health issues that fell into the medium- and low-priority groups were issues that were being adequately addressed by existing health service programs or by political, social and environmental initiatives. The categorisation of these health issues into low- or medium-priority groups did not imply they were of less significance, rather that they were of lesser prevalence or severity at that time, or were less amenable to improved health gain from alternative or more intensive interventions. Indeed, some of the health issues in the medium- or low-priority group could have been of a high priority for certain target groups, such as otitis media in Aboriginal children

The selection of high-priority health issues and the identification of effective interventions for these issues provides a sound basis for the expectation of significant health gain in the Central Sydney Area.

\section{REFERENCES}

1. MacFarlane A. Health priorities and interventions. Presentation at Health protection and promotion for children and young persons - a matter for child public health. Wuhan, China. May, 1997. Nordic School of Public Health (Gøtenborg), Hubei University (Wuhan).

2. Alperstein G, Thomson J, Crawford J. Health gain for children and youth of Central Sydney. Strategic plan. Sydney: Central Sydney Area Health Service, NSW Health Department, 1996. ISBN 0731300297.

3. Child, Adolescent and Family Health Service. Health goals and targets for Australian children and youth. Canberra: Child, Adolescent and Family Health Service and Department of Community Services and Health, Canberra, 1992.

4. Morley D. Paediatric priorities in the developing world. London: Butterworth. p. 388.

\section{TABLE 1}

\section{PRIORITY GROUPS OF HEALTH ISSUES FOR CENTRAL SYDNEY AREA HEALTH SERVICE CHILD HEALTH}

STRATEGIC PLAN

\begin{tabular}{|lll|}
\hline High priority & Medium priority & Low priority \\
\hline Tobacco-related & Youths in juvenile justice & Scoliosis \\
Related to alcohol and other substances & Sexual abuse & Autism \\
Low birthweight and prematurity & School bullying & Colour vision \\
Perinatal and infant mortality & Eating disorders & Cerebral palsy \\
Self-harm and suicide & Asthma & Developmental hip dysplasia \\
Depression & Burns and scalds & Accidental poisonings \\
At-risk behaviours (oppositional defiant & Anxiety & Psychoses \\
disorder, conduct disorder) & Falls & Congenital heart disease \\
Related to safer-sex practices & Congenital sensorineural hearing loss & Fetal alcohol syndrome \\
Physical abuse and neglect of children & Traffic-related & Iron-deficiency anaemia \\
Attention-deficit hyperactivity disorder & Postnatal depression & Drowning \\
Children of substance-abusing parents or & Sudden infant death syndrome & \\
of parents with mental illness & Post-traumatic stress disorder & \\
Excessive sunlight exposure & Fitness-related & \\
Immunisation & Lead exposure & \\
Domestic violence & Neural tube defects & \\
Learning difficulties or intelligence-related & Visual problems & \\
Nutrition-related & Conductive hearing loss & \\
Social- or environment-related & Developmental disability & \\
& Obsessive-compulsive disorder & \\
\hline
\end{tabular}

